\title{
The revised Edward Zigler Yale-Personality Questionnaire (EZPQ) for persons with intellectual disability: A new four-factor structure in confirmatory factor analysis
}

\author{
Michal Gacek $^{1}$ (D) - Lukasz Krzywoszanski ${ }^{1}$ (D) - Agnieszka Fusinska-Korpik ${ }^{2}$ \\ Published online: 22 May 2020 \\ (C) The Author(s) 2020
}

\begin{abstract}
Zigler's theory assumes that due to frequent negative personal experiences, persons with intellectual disability are likely to develop specific personality traits which hinder their functioning. The Edward Zigler-Yale Personality Questionnaire (EZPQ) is a measure that allows these traits to be assessed. However, studies in which the measure was used report problems with its factorial structure and reliability. We evaluated the psychometric properties of EZPQ in three consecutive studies conducted in three different samples of 336, 330, and 310 students with mild and moderate intellectual disability. By making successive modifications to the original version of the EZPQ, we developed and validated a revised four-factor version of the questionnaire that consists of 16 items (EZPQ-16R). The factors we identified corresponded to the following traits: readiness to explore, assignment alacrity, negative-reaction tendency, and support dependence. The reliability of the new gauge was high, and its validity was confirmed in correlational analyses with other measures of personality and adjustment. The four traits that were identified are directly derived from Zigler's theory, thus giving a new perspective on the assessment of specific personality traits in persons with intellectual disability.
\end{abstract}

Keywords Personality assessment $\cdot$ EZPQ $\cdot$ Questionnaire revision $\cdot$ Intellectual disability

\section{Background}

Zigler's $(1961,1966,1999,2001,2002)$ theory of personality development in persons with intellectual disability (ID) states that persons with ID are a group that is at risk of experiencing negative situations that lead to the development of specific personality traits which have a hindering effect on their functioning. The causes of specific personality development involve 1) a history of failure in problem-solving tasks and 2) negative social experiences. The former relates to the fact that persons with ID are more likely than persons from the general population to face cognitively demanding situations in which their abilities prove insufficient to give a correct response (Gacek et al. 2017; Bybee and Zigler 1999; Weisz 1999).

Michal Gacek

m.j.gacek@wp.pl

1 Institute of Psychology, The Pedagogical University of Krakow, Podchorazych 2, 30-084 Krakow, Poland

2 Chair of Psychiatry, The Andrzej Frycz Modrzewski Krakow University, Krakow, Poland
The latter concerns social deprivation, which in the case of persons with ID is often related to factors such as institutionalization or parental neglect (Bennett-Gates and Zigler 1999a). According to Zigler's theory, the low-quality performance of persons with ID in different types of tasks should not be explained solely in terms of intellectual deficits since certain types of behaviour related to effective investment and usage of cognitive resources by this group stem from personality characteristics rather than intellectual potency itself. In contrast to the clinical approach to personality in persons with ID that derives from psychopathology and focuses on personality disorders (Moreland et al. 2008; Torr 2003), Zigler's theory identifies traits which are not directly related to nosological categories. The described traits may also be identified in persons from the general population; however, persons with ID are more probable candidates to develop these traits as a result of the heightened risk of experiencing failure and negative social interactions.

Zigler's theory describes five personality traits which influence the performance of persons with ID (Bennett-Gates and Zigler 1999a; Zigler 2001). The first trait, called positive-reaction tendency or responsiveness, relates to a heightened need for social contact, mostly from supportive adults. The 
second trait, negative-reaction tendency, relates to initial wariness in contact and reluctance in the face of interactions with unfamiliar people. Positive- and negative-reaction tendencies are presented not as parts of a continuum but as traits which can coincide; this is because an initial fear of contact may coincide with a need for attention when a person recognizes that there are no reasons to feel threatened. The next trait, expectancy of success, relates to a person's confidence that they are able to perform well in a given task. The fourth trait, outerdirectedness, relates to the tendency to imitate others or use given cues or templates instead of attempting to solve problems using one's cognitive abilities. Lastly, effectance motivation relates to the tendency to tackle and solve problems for the sake of competence, which results in a feeling of efficacy. The significance of the mentioned traits has been confirmed in several experimental studies. When it comes to positive- and negative-reaction tendencies, it has been demonstrated that persons with ID who experienced social deprivation tend to prolong contact with adults who give them positive social reinforcements (Zigler 1961; Zigler and Balla 1972); however, such persons may also be especially cautious when it comes to contact with strangers (Harter and Zigler 1968; Bennett-Gates and Zigler 1999a). The next trait, expectancy of success, was described as low in persons with ID due to frequent experiences of failure. The results of experimental studies indicate that in the face of cognitively demanding tasks, persons with ID act as if they want to avoid failure rather than achieve complete success (Bennett-Gates and Kreitler 1999). In studies on outerdirectedness it has been reported that persons with ID have a heightened proclivity to act based on the indiscriminate usage of external cues that may be not helpful in correctly finishing a task (Bybee and Zigler 1992, 1999; Yando and Zigler 1971). Also, the results of studies on effectance motivation (Harter and Zigler 1974; BennettGates and Zigler 1999b) indicate that persons with ID often do not have a tendency to tackle problems for the sake of competence, and they generally try to avoid effort in cognitively demanding situations.

The Edward Zigler-Yale Personality Questionnaire (EZPQ) was developed to assess the specific personality traits of persons with ID that are described in Zigler's theory (Zigler et al. 2002). In the process of constructing the measure, 115 items relating to the five traits included in the theory were generated by a group of four experts in the field of intellectual disability. Then, by consensus, the items were narrowed down to the 70 which were used in the study. The authors obtained data from special education teachers regarding over 600 students with mild and moderate ID. The evaluated students had no identified organic aetiology and no secondary diagnosis. The age of the students ranged from 5 to 20 years. The 70 items used to assess the students were further narrowed down through the use of data-reduction techniques. The authors deleted the following items: those with a discrimination index less than or equal to
.30; those with a correlation of scores with the item's scale of less than .55; those with a correlation with more than one scale equal to or greater than .55 . To establish the factorial structure of the measure, the authors used principal component analyses with varimax rotation. The number of components was determined based on the examination of scree plots, eigenvalues, and factor loadings. The authors eliminated items that loaded more than one factor at a level equal to or greater than .35; items that failed to load on any one factor at a level of equal to or greater than .40 were also eliminated. The exploratory factor analysis performed on the 37 items that met the inclusion criteria did not allow the assumed five-factor structure to be obtained since several items formed two additional factors: $\mathrm{cu}$ riosity/creativity, which was linked to items related to effectance motivation; and obedience, which was linked to items related to outerdirectedness. The problem with establishing the five-factor solution might be due to the fact that the sought-after traits were experimentally studied in separate research. The operationalization of the constructs was conducted with little attention paid to the possibility that the investigated traits may overlap one another to a certain extent. Also, the experimental measures used to assess the validity of EZPQ may not be the best indicators of the studied variables since there were several significant intercorrelations between these measures and scores on EZPQ scales. The authors performed both exploratory and confirmatory factor analyses to establish the seven-factor structure of the questionnaire; however, all the factor analyses were performed on the set of data obtained from the initial sample of students. The factorial structure of the 37item version of the EZPQ was not tested by the authors on a separate sample. This seems important in terms of the large number of items reduced and the fact that the assumed fivefactor structure was changed to a seven-factor structure in the analyses.

The studies in which EZPQ has been used support the idea of perfecting this measure. In a study by Henrich et al. (2005), which was conducted on a sample of students from lowincome families, the structure of the questionnaire was not confirmed, while the internal consistency of the positivereaction tendency scale was reported as being below the acceptable level. The authors suggested a three-factor solution in which one factor would be comprised of effectance motivation, expectancy of success, and curiosity/creativity, while the two other factors would be outerdirectedness and negativereaction tendency. In a study by Gilmore and Cuskelly (2011) that was conducted on a sample of persons with Down syndrome, the reported Cronbach's coefficients were also considered by the authors as being below the acceptable level for outerdirectedness and positive-reaction tendency scales. There have also been studies in which only selected scales were used, such as obedience (Bierbaum et al. 2005), expectancy of success and effectance motivation (Mahoney et al. 2005), or positive- and negative-reaction tendency, 
obedience, and outerdirectedness (Penketh et al. 2014). In these studies, no problems with internal consistency were reported; however, the internal consistency of the separately used scales may differ significantly from the results obtained when the same items are presented in the context of other scale items (Knowles and Byers 1996; Lam et al. 2002). The EZPQ was also used in a study conducted on a sample of 14 persons with ID in which the authors suggested that addressing personality traits may help to explain the discrepancy in intelligence and adaptive behaviour test scores (Tasse and Havercamp 2006). Data on the reliability of EZPQ were not presented in this study and the reported correlations were not statistically significant due to the small sample size. Issues with the measure that arose in other studies indicate that further investigation should be conducted into the questionnaire's properties.

The purpose of the research presented in this article was to test the psychometric properties of EZPQ and to refine it based on the results of analyses performed on separate data samples. We aimed to create a short version of the questionnaire that comprises the items that best fit the tested constructs. Such an approach was justified with two arguments: firstly, filling out longer questionnaires may lead to a decrease in participants' motivation (Porter et al. 2004), especially when they need to fill out the same questionnaire a few times to assess multiple students; secondly, the reliability of personality questionnaires should increase when they contain scales that have fewer highly accurate items (Steinberg 1994).

\section{Introduction to the Present Studies}

We conducted three studies. Each study took place in special education centres in Poland over a separate schoolyear in the years 2015, 2017, and 2018. In the first study we tested the factorial structure of a direct translation of the original EZPQ; we identified and eliminated items that do not meet the accepted psychometric criteria. In the second study we used the modified version of the EZPQ, in which we retained 18 items from the original measure, and we added 17 newly developed items. Using this version of the EZPQ, we obtained a fourfactor solution that seemed to best fit the data. Finally, in the third study we confirmed the four-factor structure of EZPQ on a separate sample, and we validated a new measure that we called EZPQ-16R, in which we retained only the 16 most accurate items. This new measure that we developed in our research comprises four scales: readiness to explore, assignment alacrity, negative-reaction tendency, and support dependence. The negative-reaction tendency scale corresponds directly to the scale in the original EZPQ, while the three new scales cover the most important aspects of all the other original scales described in Zigler et al. (2002). The EZPQ-16R is not only shorter and improved in terms of its psychometric properties in comparison to the original measure, but it also taps the constructs grounded in Zigler's work in a way that advances the knowledge on the initial theoretical concepts.

\section{Inclusion Criteria for Participants}

We obtained data from special education teachers who assessed students with ID. Each teacher in each of the studies had a degree in special pedagogy, had at least two hours of classes per week with the assessed students, and had worked with them for at least six months. Students with ID were in a similar age range and fulfilled similar inclusion criteria to those used in the study by Zigler et al. (2002). As in the original EZPQ study, each time we assessed only persons with mild and moderate ID since the items used in the measure may not be suitable for assessing the functioning of persons with severe or profound disability, and we did not include persons with secondary diagnoses or organic aetiology of disability since such conditions may relate to specific behavioural profiles (Dykens 2000; Di Nuovo and Buono 2011) which could influence the results. Each student had a diagnosis of intellectual disability provided by a psychological-pedagogical counselling centre.

\section{Ethical Considerations}

The research was approved by the ethical committee at the Pedagogical University of Krakow and by the relevant school boards. The data was kept and used in accordance with the General Data Protection and Regulation Law in force (European Parliament 2016).

\section{Study 1}

The first study aimed to test the factorial structure of the direct translation of the EZPQ. We assessed a similar sample and used similar statistical analyses as described by Zigler et al. (2002).

\section{Method}

\section{Participants}

We assessed students of special education centres in Poland who fulfilled the inclusion criteria for our research. The data was obtained from special education teachers. The sample consisted of 336 students aged 8-24 $\left(M_{\text {age }}=16.90, S D=\right.$ 3.24): 191 (56.8\%) boys, 238 (70.8\%) with mild ID. 


\section{Measures}

The direct translation into Polish of the original version of the Edward Zigler-Yale Personality Questionnaire (EZPQ) (Zigler et al. 2002) was applied. The only difference was the change of the words "child" or "individual" to the word "student", which seemed more suitable for the whole range of the persons studied. We called this version of the questionnaire EZPQ-A. The measure consists of seven scales: positive- and negativereaction tendency, expectancy of success, outerdirectedness, effectance motivation, obedience, and curiosity/creativity. The student assessment is done by a teacher familiar with the typical behaviour of students. Each statement in the questionnaire is rated from 1 (very much not true) to 5 (very much true).

\section{Procedure}

We obtained three separate translations of the original EZPQ from designated experts, all of whom had degrees in Psychology or Pedagogy, were fluent in English, had authored peer-reviewed publications in this language, and had experience working with persons with intellectual disabilities. We chose the item translations considered the most accurate and a bilingual translator prepared a back-translation. We consulted the back-translation with one of the authors of the original measure, who - after making some minor changes - suggested we employ the measure in our research. After obtaining the approval of school boards, we contacted the teachers and gave them information regarding the study and instructions on how to fill in the questionnaires. The teachers completed the questionnaires at school in their free time.

\section{Statistical Analyses}

In our analyses on EZPQ-A, we aimed to test the factorial structure of the original version of this instrument. We used principal component analysis with Varimax rotation of extracted components, thus replicating the steps which led to the construction of the original measure (Zigler et al. 2002).

\section{Results}

In this study we assessed the students with EZPQ-A, which was a direct translation of the original measure. As in the study by Zigler et al. (2002), we conducted principal component analysis with Varimax rotation. The results of Bartlett's test of sphericity of correlation matrix, $\chi^{2}=6518.07, p<.001$, and the overall Kaiser-Meyer-Olkin measure of sampling adequacy, $\mathrm{MSA}=.88$, met the criteria required for such an analysis. Based on the inspection of a scree plot, six components were extracted, which accounted for $59.17 \%$ of the total variance (Component 1: 24.09\%; Component 2: 10.84\%; Component
3: 9.45\%; Component 4: 6.49\%; Component 5: $4.35 \%$; Component 6: $3.93 \%$ ). We identified items which failed to load on one component with loadings equal to or greater than .40 , and items which loaded on more than one component with loadings equal to or greater than .35 . We identified 11 such items and four additional items which loaded conceptually unrelated components. Seven of these items were attributed originally to the effectance motivation scale, three to outerdirectedness, two to positive-reaction tendency, two to expectancy of success, and one to negative-reaction tendency. The effectance motivation scale did not create a separate component in our analysis. The additional correlational analyses on the remaining scales showed that the outerdirectedness scale was positively correlated with scales that should indicate better functioning in the environment; this suggests that the items in this scale were not understood by the tested teachers in the way that was intended in Zigler's theory.

\section{Discussion}

In this study we aimed to test the factorial structure of the direct translation of the EZPQ by replicating the statistical analyses from the original study (Zigler et al. 2002). We obtained six components instead of seven, which confirms the issues with the factorial structure of the instrument that were reported in other studies (Henrich et al. 2005; Gilmore and Cuskelly 2011). Our results did not indicate the existence of the effectance motivation factor. Also, the results regarding outerdirectedness indicated that the items in this scale were not understood by the teachers as being related to negative functioning in the environment. The obtained results suggested the need to eliminate the items that did not meet the psychometric criteria. Based on our results, we decided that the measure required substantial modification before it could be used in the assessment of another sample.

\section{Study 2}

The second study aimed to test the factorial structure of the new version of the EZPQ. This version of the measure was prepared as an attempt to operationalize anew the seven-factor structure of the questionnaire by changing the initial set of items based on the results of Study 1 .

\section{Method}

\section{Participants}

We assessed the students of special education centres in Poland who fulfilled the inclusion criteria for our research. 
Table 1 Items used in different versions of EZPQ

EZPQ-16R Items reduced after analyses

of EZPQ-B and EZPQ-C

Readiness to explore Uses material in imaginative ways $(\mathrm{CC})^{*}$

$(\mathrm{CC}+\mathrm{ES})$

Is creative $(\mathrm{CC})^{*}$

Willingly accepts challenges,

even not knowing if he/she can deal with them (ES)

Likes challenges (ES)

Assignment alacrity $(\mathrm{EM}+\mathrm{OB})$

Likes to perform tasks that are given, even when no reward is expected (EM) Eagerly gets down to work (EM) Does not listen to rules $(-)(\mathrm{OB})^{*}$ Usually does as told (OB) * Negative-reaction
tendency (NR)

Support dependence $(\mathrm{OD}+\mathrm{PR})$
Spends more time alone (NR) *

Tends to withdraw and isolate him/herself when supposed to be in a group (NR) *

Isolates him/herself (NR) *

Usually doesn't trust others (NR) ${ }^{*}$

Seeks others' help, even when he/she could solve the task by him/herself (OD)

Expects clues from others even when they are not necessary (OD)

Is constantly seeking attention and praise (PR) *

Wants adult help even when it's not really needed (PR) ${ }^{*}$
Shows curiosity about many things (CC) * B

Is interested in things that happen around him/her $(\mathrm{CC})^{\mathrm{B}}$

Is inventive and unconventional while performing tasks $(\mathrm{CC})^{\mathrm{C}}$

Is confident (ES) * B

Is sure things will work out well when he/she has trouble solving a problem at school (ES) ${ }^{*} \mathrm{~B}$

Is sure things will work out well when he/she has new work to do at school (ES) ${ }^{* \mathrm{C}}$

Persistently tries to finish tasks that are given $(\mathrm{EM})^{\mathrm{C}}$

Gives up on a task before it is finished (-)

$(\mathrm{EM})^{\mathrm{B}}$

Following task commencement, he/she works by him/herself and does not require further encouragement $(\mathrm{EM})^{\mathrm{B}}$

Accepts imposed limits $(\mathrm{OB})^{*} \mathrm{~B}$

Is disobedient; doesn't mind well $(-)(\mathrm{OB}){ }^{*} \mathrm{~B}$

Is able to submit to the rules in force $(\mathrm{OB})^{\mathrm{C}}$

Tends to keep thoughts, feelings, or products to him/herself (NR) ${ }^{*} \mathrm{C}$

Prefers to imitate others' work rather than solve the problem by him/herself (OD) ${ }^{\text {B }}$

Prefers to follow the example of others than try and solve the problem by him/herself $(\mathrm{OD})^{\text {B }}$

Repeats others' judgments, not thinking them through; treats them as if they were his/hers (OD) ${ }^{\mathrm{C}}$

Works harder during class when an adult's attention is focused on him/her (PR) ${ }^{\mathrm{B}}$

Stops performing a task if not given attention by an adult $(\mathrm{PR}){ }^{\mathrm{C}}$

Seems to prefer adults to children (PR) ${ }^{*} \mathrm{~B}$

${ }^{\mathrm{B}}$ item used in EZPQ-B and reduced; ${ }^{\mathrm{C}}$ item used in EZPQ-C and reduced; ${ }^{*}$ item taken from the original version of the EZPQ; $(-)$ item reversely scored. The abbreviations describe the scales from the original EZPQ: $\mathrm{CC}=$ curiosity/creativity, $\mathrm{ES}=$ expectancy of success, $\mathrm{EM}=$ effectance motivation, $\mathrm{OB}=$ obedience, $\mathrm{NR}=$ negative-reaction tendency, $\mathrm{OD}=$ outerdirectedness, $\mathrm{PR}=$ positive-reaction tendency

Again, the data was obtained from special education teachers. The sample consisted of 330 students aged 7-23 $\left(M_{\text {age }}=\right.$ $16.52, S D=3.01): 189$ boys $(57.3 \%), 247(74.8 \%)$ with mild ID.

\section{Measures}

EZPQ-B, the modified Polish version of EZPQ, was used in this study. This measure consisted of 35 items, of which 18 were also used in the original measure, while 17 were newly developed (see Table 1). Each of the seven scales contained five items. We added one new item to the obedience scale, and two new items to the curiosity/creativity, expectancy of success, and positive-reaction tendency scales. Also, we replaced all items for the effectance motivation and outerdirectedness scales with new ones. The former scale did not manifest as a separate component in Study 1; in the case of the latter scale, we intended to make it clear that the items relate to situations in which a student acts on cues and templates despite having the ability to perform well without such guidance. The negative-reaction tendency scale was the only one which contained five items from the original measure.

\section{Procedure}

We asked three designated experts to prepare a set of items for each of the seven scales in EZPQ-B. All the experts had degrees in Psychology or Pedagogy, had published papers related to their field, and had experience working with persons with intellectual disabilities. Each expert was presented with a definition of the sought-after constructs and the set of original items which formed each of the scales in EZPQ-A. The experts proposed the new items that in their opinion would assess the constructs most accurately. All the propositions were compared and discussed by the authors of this article. The items that were considered the most accurate and clearly 
phrased were added to EZPQ-B. After obtaining the approval of school boards, we contacted the teachers and gave them information regarding the study and instructions on how to fill in the questionnaires. The teachers completed the questionnaires at school in their free time.

\section{Statistical Analyses}

In this study we used EZPQ-B. We performed principal component analysis, this time using Oblimin rotation, which allows components to be intercorrelated (Pedhazur and Schmelkin 1991; Field et al. 2012). Oblique rotation was used since - according to Zigler's theory - the tested constructs should be seen as correlated and not orthogonal.

\section{Results}

Based on the results obtained in Study 1, we constructed EZPQ-B, which was used to assess the students in this study. To explore the factor structure of EZPQ-B, principal component analysis with Oblimin rotation was performed. The results of Bartlett's test of sphericity, $\chi^{2}=$ $7533.30, p<.001, \mathrm{MSA}=.90$, met the criteria for principal component analysis. For this sample we also calculated MSA values for individual items. The obtained scores ranged from .75 to .95 . Based on both the scree plot and the parallel analysis, four components were extracted. These four components explained $59.08 \%$ of the variance (Component 1: 19.48\%, Component 2: 16.28\%, Component 3: $13.23 \%$, Component 4: 10.08\%). The first component in the analysis consisted of the items that were attributed to the curiosity/creativity and expectancy of success scales. When analysing the items' content, we called this component 'readiness to explore'. The second component consisted of the items from the effectance motivation and obedience scales. We called this component 'assignment alacrity'. The third component included negative-reaction tendency items. The fourth component consisted of the items attributed to the outerdirectedness and positive-reaction tendency scales; this component was called 'support dependence'.

In the next step we analysed the properties of the items. We identified the factor loadings with a value above 40 . Three items loaded two different components and one item loaded a conceptually unrelated component. We removed these items and obtained the corrected item-total correlations for the remaining items. This allowed us to obtain information which we used to modify the measure for use in the next study (see Fig. 1.)

\section{Discussion}

In this study we attempted to test the factorial structure of EZPQ-B, which is a modified version of the original measure. As in Study 1, we did not obtain results that would indicate the assumed seven-factor solution. The difficulties in assessing the original constructs may be explained by the fact that the empirical background of Zigler's theory constitutes results from separate experimental studies, and the question of how the tested traits relate to one another was not thoroughly answered (Zigler 1999, 2001). In this study we identified four components which are related to traits derived from the original concepts in Zigler's theory. We labelled these components as readiness to explore, assignment alacrity, negative-reaction tendency, and support dependence. The negative-reaction tendency component overlaps with the items that make up the original EZPQ negative-reaction-tendency scale. This aspect of personality in persons with ID relates to initial reluctance in contact and difficulties in social interactions with strangers (Bennett-Gates and Zigler 1999a). Each of the other three components we obtained was related to two separate traits from Zigler's theory. The component labelled "readiness to explore" covered the content of two of the original EZPQ scales: expectancy of success and curiosity/creativity (Bennett-Gates and Kreitler 1999). We identified this personality trait as being related to the tendency to actively explore one's environment and not withdraw from engagement in challenging situations. The assignment alacrity component was composed of items from the obedience and effectance motivation scales (Harter and Zigler 1974; Bennett-Gates and Zigler 1999b). This trait relates to following rules and guidelines and finding pleasure in assigned tasks. Finally, the supportdependence component consisted of items from the positive-reaction-tendency and outerdirectedness scales (Bennett-Gates and Zigler 1999a; Bybee and Zigler 1992, 1999). This trait relates to the need for supportive contact and the tendency to use external cues in challenging situations. Based on the results of the second study, we decided to once again modify the measure that we used and to test the four-factor solution in a subsequent study conducted on a separate sample.

\section{Study 3}

In this study we aimed to confirm the assumed four-factor structure of specific personality traits in persons with ID and to refine the modified EZPQ into a short questionnaire with balanced item content. We also intended to test the reliability and validity of the scales of the refined version of the questionnaire. 
Fig. 1 Diagram presenting classification and selection of EZPQ-B items in study 2. based on values of component loadings in PCA and corrected item-total correlations
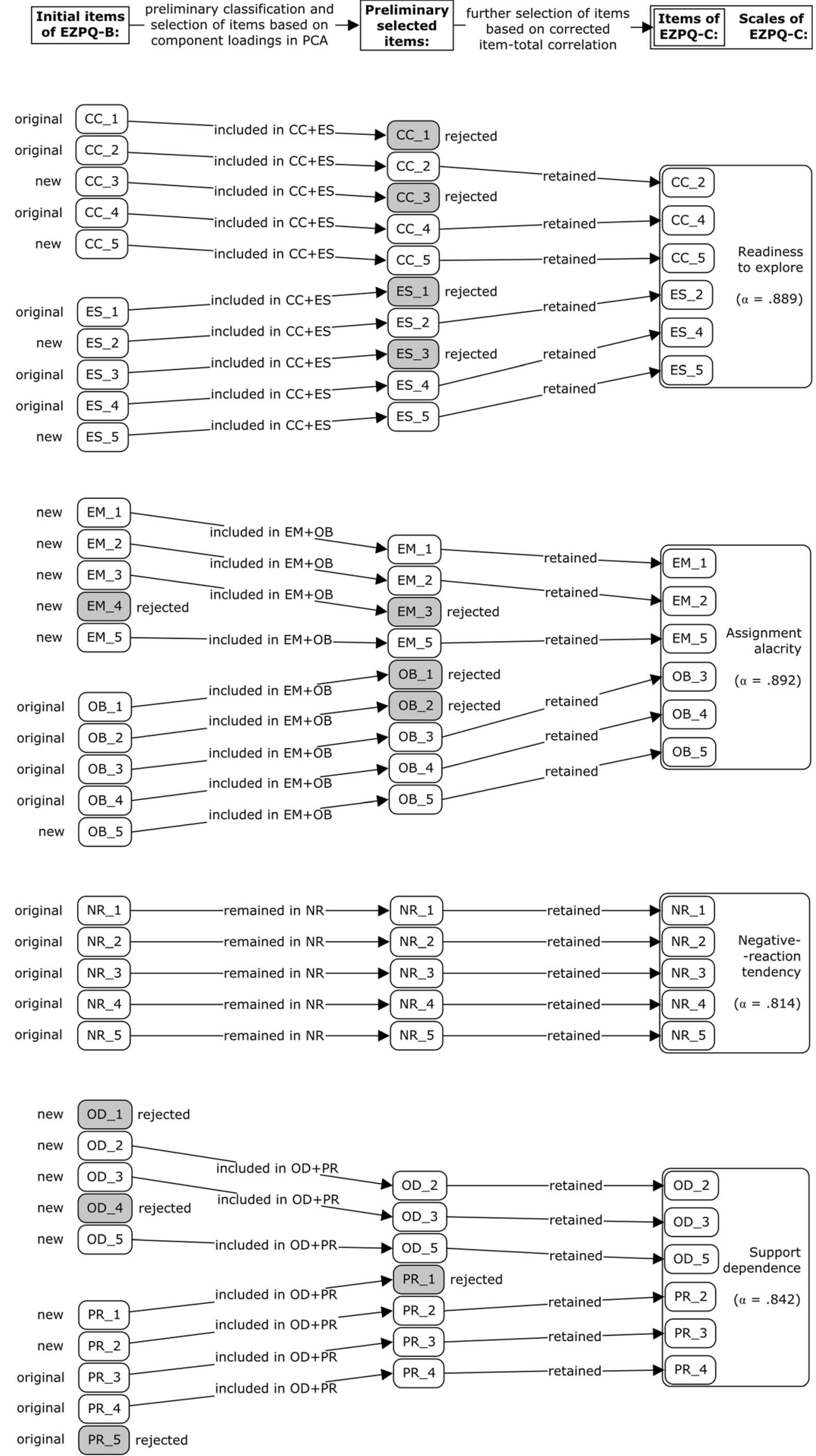


\section{Method}

\section{Participants}

As in the two previously described studies, we assessed the students of special education centres who met the inclusion criteria. The data was obtained from special education teachers. The sample consisted of 310 students aged 9-24 $\left(M_{\text {age }}=16.56, S D=3.84\right): 175(56.5 \%)$ boys, $227(73.2 \%)$ with mild ID.

\section{Measures}

The Edward Zigler-Yale Personality Questionnaire (EZPQ) was used in the modified version, which we called EZPQ-C. In order to prepare the instrument with balanced content related to the original constructs, we used the information obtained in Study 2. From the items that were included in EZPQ-B, we selected six items for each of the new components and three items from each of the scales with the highest item-total correlation coefficients. Five items were used for the negativereaction tendency scale. This version of the measure consisted of 23 items (see Fig. 1 for the data-reduction procedure and Table 1 for the item content). EZPQ-C was prepared with the assumption that the model which gives the best data fit should comprise the four personality traits which came up as separate components in our analyses in Study 2. We left the original negative-reaction tendency trait and labelled the new scales as readiness to explore, assignment alacrity, and support dependence. In this approach, readiness to explore comprises items that formed the curiosity/creativity and expectancy of success scales and is related to the general tendency to actively explore one's environment and engage in actions by testing different possibilities. Assignment alacrity comprises items related initially to effectance motivation and obedience. This trait relates to the tendency to follow proper social guidelines, such as instructions in class, and to enjoy doing work that is assigned. Support dependence is comprised of the positivereaction tendency and the outerdirectedness items and is related to the tendency to seek supportive contact with adults and to relate to and to seek external cues and templates when faced with challenging situations. Better environmental adjustment should be indicated by higher results in the readiness-toexplore and assignment alacrity tendencies and lower results in the support-dependence and negative-reaction tendencies. The revised version of the questionnaire which we established in the confirmatory factor analysis comprised 16 items and was named EZPQ-16R.

Big Five Questionnaire - Children (BFQ-C). BFQ-C (Barbaranelli et al. 2003), adapted into Polish by Cieciuch et al. (2016), is a 65-item questionnaire that allows the Big Five personality traits to be tested in children and adolescents. We used the version of the questionnaire which allows assessment of traits by a third party, e.g. a parent or teacher who is familiar with a child's typical behaviour. The tested traits are energy/extraversion, agreeableness, conscientiousness, emotional instability, and intellect/ openness. The energy/extraversion scale concerns being enthusiastic, assertive, and self-confident while performing a variety of activities. Agreeableness concerns being sensitive to the needs and concerns of others. Conscientiousness is related to being orderly and precise in fulfilling commitments and to a person's dependability. Emotional instability relates to the tendency to experience feelings of anger, depression, and anxiety. Finally, the intellect/openness trait taps the broadness of cultural interests, self-reported creativity, fantasy, intellect, and interest in other people. The statements in the questionnaire are rated on a 1-to-5 Likert-type scale. Since the measure had not been used previously to assess students with ID, we added an answer box marked "no information", which teachers could mark when they felt they had no information regarding certain behaviours. The Polish version of the BFQ-C has high reliability and satisfactory properties when it comes to convergent, discriminant, and external validity (Cieciuch et al. 2016).

Strengths and Difficulties Questionnaire (SDQ). The SDQ (Goodman 1997, 2001) is a 25-item measure which allows the assessment of children's and adolescents' attributes in five domains: emotional symptoms, conduct problems, hyperactivity-inattention, peer problems, and prosocial behaviour. The four scales that measure difficulties can be summed to generate a total difficulties score. The statements are rated on a 3point Likert scale. In our study we used a version of the scale which allows third-party assessment. The measure has satisfactory psychometric properties that have been investigated in numerous cross-cultural studies (for a review see Stone et al. 2010) and have also been used and validated in Poland (see the official site http://www.sdqinfo.com/ or, for example, Skoczeń et al. 2018).

\section{Procedure}

After obtaining the approval of school boards, we contacted the teachers and gave them information regarding the study and instructions on how to fill in the questionnaires. The teachers completed the questionnaires at school in their free time. Teachers were asked to fill in EZPQ-C and either BFQ$\mathrm{C}$ or SDQ (assigned randomly). Retest data on the final version of EZPQ was obtained after a month and then after three months at one of the centres in which the study took place.

\section{Statistical Analyses}

To verify the four-factor structure of the shortened version of the EZPQ, we performed confirmatory factor analyses (CFA) for responses given to the 23 items of EZPQ-C. The CFA was 
preceded by an analysis of the Kaiser-Meyer-Olkin measure of sampling adequacy to identify and omit any items not sufficiently related to other items. Then we specified a fourcorrelated-factors CFA model using the lavaan R Package for Structural Equation Modelling (Rosseel 2012), implemented in JASP open-source statistical program (JASP Team 2019). Since the distribution of responses to EZPQ-C items deviated from normality, the Diagonally Weighted Least Squares (DWLS) estimation method was used. Chisquare to df ratio $\left(\chi^{2} / \mathrm{df}\right)$, Root Mean Square Error of Approximation (RMSEA), Standardized Root Mean Square Residual (SRMR), Bentler's Comparative Fit Index (CFI), Tucker-Lewis Index (TLI) and Bentler-Bonett Normed Fit Index (NFI) were used to evaluate the overall model fit. Then we refined the initial four-factor CFA model by eliminating items with factor loadings that were too low from their parental scales. In the next steps we further modified the model by eliminating items with relevant associations with the extrinsic factor, and we dealt with the mutual correlations of error terms by eliminating items from the CFA model or by allowing the residuals of two items to be correlated according to the modification indices. In the next step we analysed the corrected item-total correlations between the remaining items and the scales covering them, and we removed the items with the lowest values to obtain scales of equal length and balanced content. The responses to the items selected in this way were entered into the final CFA model, which included the EZPQ16R items. The scales of EZPQ-16R were then tested for their reliability and convergent validity. We used Cronbach's $\alpha$ as a measure of internal consistency, and test-retest Pearson correlations as a measure of temporal stability of the scores in EZPQ-16R scales. Also, we correlated scores in the EZPQ$16 \mathrm{R}$ scales with the two other measures used in the study.

\section{Results}

The overall Kaiser-Meyer-Olkin measure of sampling adequacy for responses given to items of EZPQ-C (MSA = .90) met the criterion for the subsequent factor analyses. The MSA values for responses to most of the individual items ranged from .79 to .95 . The only exception was one item from the negative-reaction tendency scale that had a value of .70 and was therefore not included in further analyses. The initial CFA model which included 22 items had an acceptable fit, $\chi^{2} / \mathrm{df}=$ 2.44, RMSEA $=.068,90 \% \mathrm{CI}=.061-.076$, SRMR $=.091$, $\mathrm{CFI}=.958, \mathrm{TLI}=.952, \mathrm{NFI}=.931 . \mathrm{In}$ the next step we analysed the model parameters in detail. To improve the fit of the model, we removed one item from the supportdependence scale which had a low factor loading of .30 and an error term of .91. The CFA specified without this item yielded a high modification index value, 93.5, for the path from the assigned alacrity factor to one of the support dependence items. When specifying the model without this item, we obtained the correlation of error terms for two items from assignment alacrity. We removed the item with the lowest standardized path coefficient value. After the steps we took to improve the model, we obtained an unequal number of items for the factors. Thus, we removed the three items with the lowest corrected item-total correlation coefficients among the components of a given scale to balance the length and content of the developed scales. Again, we selected an equal number of items related to the original constructs for each of the factors. The final model we tested comprised 16 items belonging to four scales (see Fig. 2 for the procedure and values of standardised path coefficients; see Table 1 for the content of the items). This model had a good fit, $\chi^{2} / \mathrm{df}=1.80$, RMSEA $=.051$ with $90 \%$ CI from .039 to .063 , $\mathrm{SRMR}=.074, \mathrm{CFI}=.979, \mathrm{TLI}=.974, \mathrm{NFI}=.954$, and all inspected fit indexes were improved in comparison to the initial model (Hu and Bentler 1999). As a result of the analyses, we developed the improved four-factor version of the original questionnaire: EZPQ-16R.

The descriptive statistics for EZPQ-16R are presented in Table 2. All the intercorrelations between the four scales were significant at $p<.001$, and the correlation coefficients ranged from low to moderate. As expected, the scales with higher scores that indicated better environmental adjustment correlated negatively with the scales in which higher scores should indicate problems with adjustment. The Cronbach's $\alpha$ coefficients were high for all the scales: .87 for readiness to explore, .84 for assignment alacrity, .84 for negative-reaction tendency, and .84 for support dependence. The corrected item-rest correlations ranged from .68 to .76 for readiness to explore, .62 to .74 for assignment alacrity, .61 to .80 for negative-reaction tendency, and .51 to .77 for support dependence. Test-retest correlations performed after a month were high, ranging from .72 to .82 , and after three months from .61 to .87 (see Table 2).

Pearson's linear correlation coefficient was used to assess the relationships between scores in EZPQ-16R scales and scores in BFQ-C scales. Spearman's rank-order correlation coefficient was used to determine the relationships between scores in EZPQ-16R scales and scores in SDQ scales since the distributions of scores of SDQ scales were skewed. The correlations of EZPQ-16R and BFQ-C were significant for the scales that were expected to share certain facets related to the tested constructs. The scales in EZPQ-16R, in which higher scores should indicate better adjustment, were positively correlated with the scores in SDQ scales which also indicate better adjustment; on the other hand, they were negatively correlated with SDQ scales which indicate difficulties. In the case of EZPQ-16R scales, in which higher scores should indicate difficulties in adjustment, the pattern of correlation with the scores in the SDQ scales was the opposite. The values of correlation coefficients are presented in Table 3. 
Fig. 2 Diagram presenting the structure and standardised path coefficients for the final four correlated factors model of EZPQ-16R obtained in CFA performed on the results of Study 3
Results of CFA for final measurement model of EZPQ-16R scales in study 3.

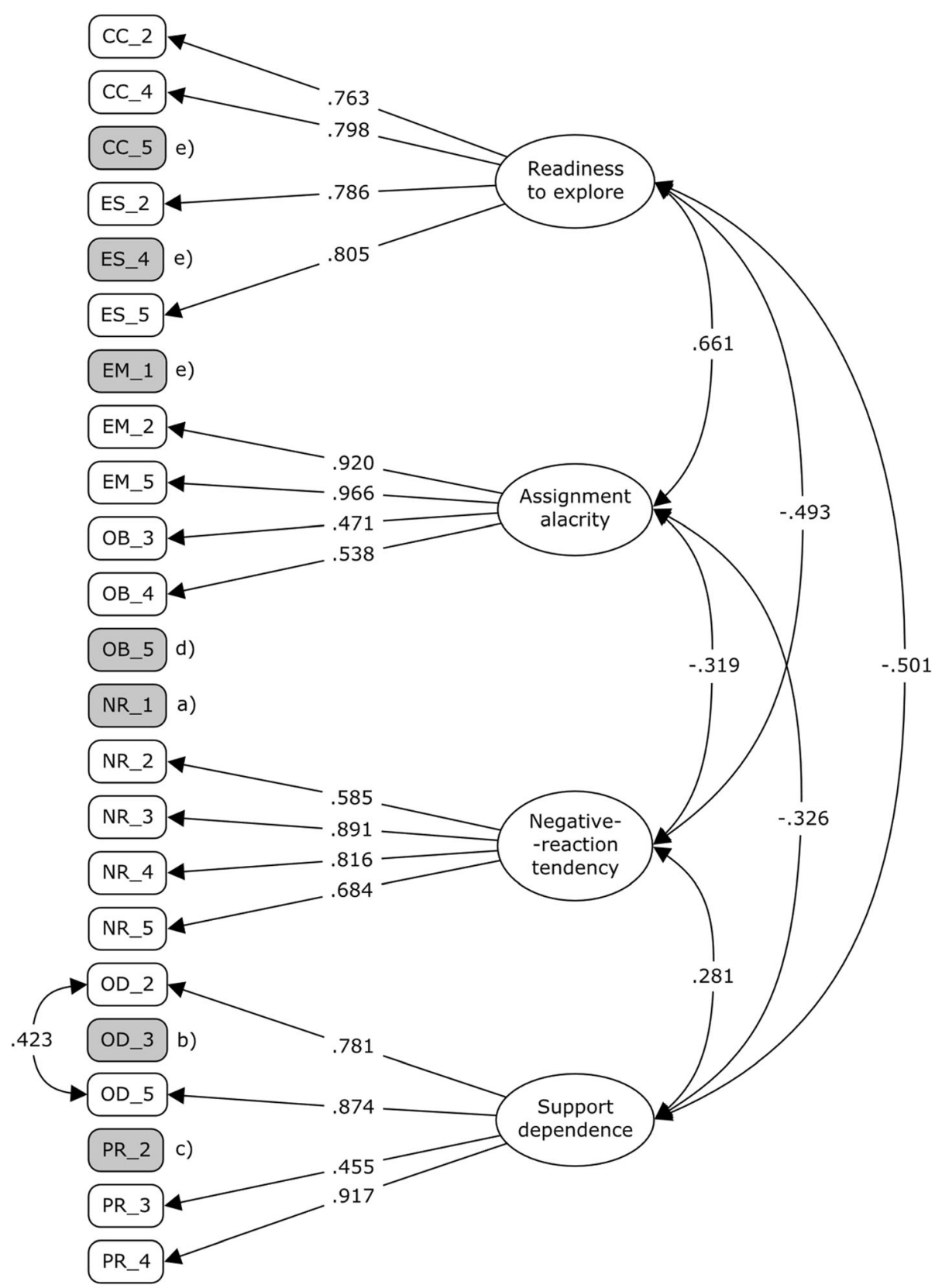

Note: items marked in grey were removed in the following five steps:

a) NR_1 was excluded from the CFA model due to a low measure of sampling adequacy (MSA) value in the initial Kaiser-Meyer-Olkin analysis.

b) OD_3 was removed from the CFA model due to low loading for the path from its parental factor (Support dependence).

c) PR_2 was removed from the CFA model due to high loading for the path from the extrinsic factor (Assignment alacrity), disclosed by high modification index value.

d) OB 5 was removed from the CFA model due to strong intercorrelation of its residual with the residual of OD_5, as indicated by the high modification index value.

e) CC_5, ES_4, and EM_1 were removed due to the low values of the corrected item total correlation, as obtained in the analysis of internal consistency of EZPQ-R scales. 


\section{Discussion}

In our third study we aimed to confirm the assumed fourfactor structure of the version of the EZPQ questionnaire which comprised items obtained in Study 2. We attempted to refine this measure into a short four-scale questionnaire and to determine the basic psychometric properties of its measurement scales. The results of our initial CFA suggested that the four-factor model of personality showed a fairly good overall fit with the results obtained in our third study, but further elimination of certain items based on values of path coefficients and modification indices was required. Accordingly, in the next steps, three items were removed from the structural model and correlation between the error terms of two outerdirectedness items was allowed. Then the next three items with the lowest values of the corrected item-total correlation on the given scale were eliminated. As a result, 16 items were retained, four in each of the scales. The final model, which comprised 16 items linked to four corresponding correlated factors, showed a good overall fit and the values of the fit measures obtained for it improved in comparison to the initial model. Values for both the path coefficients for correlations between the factors in the final CFA model and Pearson correlations between EZPQ-16R scales showed that four factors of the simplified model of the personality of persons with ID we have developed are unorthogonal to each other. Scores in the readiness-to-explore scale are positively correlated with scores in the assignment alacrity scale; they are negatively correlated with scores in the negative-reaction-tendency and support-dependence scales, while the two latter scales are positively correlated with each other but negatively correlated with the two former. As expected, the two scales which measure adaptive aspects of personality and the two scales which measure personality aspects related to difficulties in functioning in the environment in persons with ID are negatively associated with each other. The demonstrated directions of the relationships between personality dimensions are in line with expectations based on Zigler's theory, which confirms the theoretical and factorial validity of the EZPQ-16R that we have developed.

The EZPQ-16R was tested for reliability and convergent validity. When it comes to reliability, both the internal consistency and the temporal stability of the four scales were on a similar level as in the original study, in which the internal consistency of the scales ranged from .76 to .91; retest correlations performed after three months ranged from .65 to .94 (Zigler et al. 2002). The convergent validity of EZPQ-16R was assessed based on the results of correlational analyses with the scores in the BFQ-C and SDQ scales. For BFQ-C, the readiness-to-explore scale correlated highest with energy/extraversion and intellect/ openness. Each of these three constructs may be seen as related to the tendency to engage in new activities and explore the environment. The assignment alacrity scale had the highest correlations with conscientiousness and agreeableness, which are the two constructs that include behaviours related to obedience and continuous engagement in given tasks. The negative-reaction tendency was related negatively to energy/extraversion, and the supportdependence scale had the highest negative correlation with intellect/openness. In the case of the negative-reaction tendency scale, the tendency to avoid contact should be related to more introverted behaviour; when it comes to support dependence, the tendency to seek help from others should be related to difficulties in engaging in intellectual activities under a person's own volition. In SDQ, the score in the total difficulties scale was correlated positively with the scores in the EZPQ-16R scales that indicate problems with adjustment; it was negatively correlated with the scales that indicate proper adjustment. The opposite pattern of correlations was obtained with the prosocial scale in SDQ, thus further supporting the validity of EZPQ-16R.

Table 2 Descriptive statistics and correlations for EZPQ16-R

\begin{tabular}{|c|c|c|c|c|c|c|c|c|}
\hline & \multicolumn{3}{|c|}{ Descriptive statistics } & \multicolumn{2}{|c|}{ Retest correlations } & \multicolumn{3}{|c|}{ Intercorrelations } \\
\hline & Mean (SD) & Skew & Kurtosis & $\begin{array}{l}1 \text { month } \\
(n=53)\end{array}$ & $\begin{array}{l}3 \text { months } \\
(\mathrm{n}=53)\end{array}$ & $\begin{array}{l}\text { Readiness to } \\
\text { explore }\end{array}$ & $\begin{array}{l}\text { Assignment } \\
\text { alacrity }\end{array}$ & $\begin{array}{l}\text { Negative-reaction } \\
\text { tendency }\end{array}$ \\
\hline Readiness to explore & $11.3(3.51)$ & .141 & -.349 & .82 & .82 & & & \\
\hline Assignment alacrity & $13.59(3.4)$ & -.168 & -.527 & .74 & 1.79 & .53 & & \\
\hline $\begin{array}{r}\text { Negative-reaction } \\
\text { tendency }\end{array}$ & $9.88(3.39)$ & .854 & .456 & .82 & .87 & -.40 & -.22 & \\
\hline Support dependence & $12.2(3.48)$ & .381 & -.579 & .72 & .61 & -.39 & -.27 & .21 \\
\hline
\end{tabular}

$p<.001$ for all the correlations presented in the table 
Table 3 Correlations of EZPQ16-R scales with BFQ-C and SDQ scales

\begin{tabular}{|c|c|c|c|c|c|}
\hline & & \multicolumn{4}{|l|}{ EZPQ-16R } \\
\hline & & Readiness to explore & Assignment alacrity & Negative-reaction tendency & Support dependence \\
\hline \multirow[t]{5}{*}{ BFQ-C } & Energy/extraversion & $.52 * *$ & .18 & $-.45 * *$ & -.18 \\
\hline & Emotional instability & -.13 & $-.48 * *$ & -.04 & $.25^{*}$ \\
\hline & Intellect/openness & $.55^{* *}$ & $.29 *$ & -.01 & $-.36^{*}$ \\
\hline & Conscientiousness & $.48 * *$ & $.77 * *$ & -.06 & $-.25 *$ \\
\hline & Agreeableness & $.38 *$ & $.64 * *$ & -.21 & -.11 \\
\hline \multirow[t]{6}{*}{ SDQ } & Emotional symptoms & $-.15 *$ & $-.16^{*}$ & $.39 * *$ & $.29 * *$ \\
\hline & Conduct problems & $-.14 *$ & $-.55^{* *}$ & .08 & .06 \\
\hline & Hyperactivity/inattention & $-.35 * *$ & $-.62 * *$ & .09 & $.35 * *$ \\
\hline & Peer problems & $-.36 * *$ & $-.23 *$ & $.69 * *$ & $.26^{* *}$ \\
\hline & Prosocial & $.45 * *$ & $.58 * *$ & $-.31 * *$ & $-.20 *$ \\
\hline & Total difficulties & $-.37 * *$ & $-.57 * *$ & $.44 * *$ & $.36^{* *}$ \\
\hline
\end{tabular}

$* * p<.001, * p<.05$

\section{Final Remarks}

In our research we aimed to test the factorial structure of EZPQ and to refine it into a short questionnaire based on the results of analyses performed on three separate data samples. We developed a measure that we called EZPQ-16R, which allows the assessment of four traits instead of the seven that were included in the questionnaire prepared by Zigler et al. (2002). EZPQ-16R retains the original negative-reaction tendency trait and introduces three new ones: readiness to explore, assignment alacrity, and support dependence. Each of the new traits covers some of the aspects of the original constructs. Readiness to explore covers the aspects of curiosity/ creativity and expectancy of success (Bennett-Gates and Kreitler 1999) and is related to the tendency to actively explore the environment and engage in actions by testing different possibilities. Assignment alacrity comprises the items that were initially attributed to the effectance motivation and obedience scales (Harter and Zigler 1974; Bennett-Gates and Zigler 1999b). This trait relates to the tendency to follow social rules and guidelines and to derive pleasure from assigned work. Support dependence covers the aspects of positivereaction tendency and outerdirectedness (Bennett-Gates and Zigler 1999a; Bybee and Zigler 1992, 1999) and is related to the tendency to seek supportive contact with adults and to relate to external cues in challenging situations. The EZPQ$16 \mathrm{R}$ presents a new approach to the measurement of specific personality traits in persons with ID that is grounded in Zigler's theory of personality.

Our research has several limitations. First, we did not test the criterion validity of the new measure. In future, the use of experimental tasks or other external indicators of functioning in the environment should give information on the relation between the assessed traits and a person's actual behaviour.
Second, it is important to note that the measure relies on assessment by a third party. We obtained information only from teachers who had experience working with the assessed students. In further research, it also seems important to obtain information from other persons such as parents or caregivers to see if the traits are recognized outside the educational context. Third, we used the measure only in groups of persons with ID. Using the measure to assess typically developing children and comparing the results with those obtained by persons with ID should give additional information regarding the specificity of personality development in those with ID. Fourth, in our study we did not include persons with genetic syndromes. Gathering information on such persons should help to answer questions regarding the relationship between the genetic causes of disability and the tendency to develop certain personality traits. Also, including other variables such as periods of institutionalization or family characteristics should further allow explanation of the exact reasons that these traits appear and the things they can be correlated with.

\section{Conclusions}

In our research we developed a short measure called EZPQ16R, which comprises four scales: readiness to explore, assignment alacrity, negative-reaction tendency, and support dependence. The measure has good psychometric properties and can be used to assess personality traits in persons with ID. To increase reliability, we used only the most accurate items, which were selected based on stringent criteria. We also further advanced the knowledge on the factorial structure of the specific personality traits described in Zigler's $(1999,2001)$ theory by proposing new traits which include essential aspects of the original constructs. The EZPQ-16R is a valid and 
reliable measure which can be used both in academic studies as well as in educational and clinical contexts.

Funding Financial support was provided by the Polish Ministry of Science and Higher Education from funds for statutory research at the Pedagogical University of Krakow.

\section{Compliance with Ethical Standards}

Declarations of Interest On behalf of all the authors, the corresponding author states that there is no conflict of interest.

Open Access This article is licensed under a Creative Commons Attribution 4.0 International License, which permits use, sharing, adaptation, distribution and reproduction in any medium or format, as long as you give appropriate credit to the original author(s) and the source, provide a link to the Creative Commons licence, and indicate if changes were made. The images or other third party material in this article are included in the article's Creative Commons licence, unless indicated otherwise in a credit line to the material. If material is not included in the article's Creative Commons licence and your intended use is not permitted by statutory regulation or exceeds the permitted use, you will need to obtain permission directly from the copyright holder. To view a copy of this licence, visit http://creativecommons.org/licenses/by/4.0/.

\section{References}

Barbaranelli, C., Caprara, G. V., Rabasca, A., \& Pastorelli, C. (2003). A questionnaire for measuring the big five in late childhood. Personality and Individual Differences, 34(4), 645-664.

Bennett-Gates, D., \& Kreitler, S. (1999). Expectancy of success in individuals with mental retardation. In E. Zigler \& D. BennettGates (Eds.), Personality development in individuals with mental retardation (pp. 130-144). Cambridge: Cambridge University Press.

Bennett-Gates, D., \& Zigler, E. (1999a). Motivation for social reinforcement: Positive- and negative-reaction tendencies. In E. Zigler \& D. Bennett-Gates (Eds.), Personality development in individuals with mental retardation (pp. 107-129). Cambridge: Cambridge University Press.

Bennett-Gates, D., \& Zigler, E. (1999b). Effectance motivation and the performance of individuals with mental retardation. In E. Zigler \& D. Bennett-Gates (Eds.), Personality development in individuals with mental retardation (pp. 145-164). Cambridge: Cambridge University Press.

Bierbaum, L. J., Henrich, C. C., \& Zigler, E. F. (2005). Disobedient behaviours in children with intellectual disability. Journal of Intellectual \& Developmental Disability, 30(2), 115-119.

Bybee, J., \& Zigler, E. (1992). Is outerdirectedness employed in a harmful or beneficial manner by students with and without mental retardation? American Journal on Mental Retardation, 96(5), 512-521.

Bybee, J., \& Zigler, E. (1999). Outerdirectedness in individuals with and without mental retardation: A review. In E. Zigler \& D. Bennett-Gates (Eds.), Personality development in individuals with mental retardation (pp. 165-205). Cambridge: Cambridge University Press.

Cieciuch, J., Toczyłowska-Niemiec, K., \& Barbaranelli, C. (2016). Kwestionariuszowy pomiar pięciu cech osobowości dzieci i dorastających. Polska adaptacja big five questionnaire-children (BFQ-C) [questionnaire measurement of five personality traits in children and adolescents: A polish adaptation of big five questionnaire-children (BFQ-C)]. Psychologia Rozwojowa, 21(2), 73-85.

Di Nuovo, S., \& Buono, S. (2011). Behavioral phenotypes of genetic syndromes with intellectual disability: Comparison of adaptive profiles. Psychiatry Research, 189(3), 440-445.

Dykens, E. M. (2000). Annotation: Psychopathology in children with intellectual disability. The Journal of Child Psychology and Psychiatry and Allied Disciplines, 41(4), 407-417.

European Parliament (2016). Regulation (EU) 2016/679 of the European Parliament and of the Council of 27 April 2016 on the protection of natural persons with regard to the processing of personal data and on the free movement of such data, and repealing Directive 95/46/EC (General Data Protection Regulation) http://data.europa.eu/eli/reg/ 2016/679/oj

Field, A., Miles, J., \& Field, Z. (2012). Discovering statistics using R. London: Sage Publications Ltd..

Gacek, M., Smoleń, T., \& Pilecka, W. (2017). Consequences of learned helplessness and recognition of the state of cognitive exhaustion in persons with mild intellectual disability. Advances in Cognitive Psychology, 13(1), 42-51.

Gilmore, L., \& Cuskelly, M. (2011). Observational assessment and maternal reports of motivation in children and adolescents with down syndrome. American Journal on Intellectual and Developmental Disabilities, 116(2), 153-164.

Goodman, R. (1997). The strengths and difficulties questionnaire: A research note. Journal of Child Psychology and Psychiatry, 38(5), 581-586.

Goodman, R. (2001). Psychometric properties of the strengths and difficulties questionnaire. Journal of the American Academy of Child \& Adolescent Psychiatry, 40(11), 1337-1345.

Harter, S., \& Zigler, E. (1968). Effectiveness of adult and peer reinforcement on the performance of institutionalized and noninstitutionalized retardates. Journal of Abnormal Psychology, 73(2), 144-149.

Harter, S., \& Zigler, E. (1974). The assessment of effectance motivation in normal and retarded children. Developmental Psychology, 10(2), 169-180.

Henrich, C. C., Wheeler, C. M., \& Zigler, E. (2005). Motivation as a facet of school readiness in a head start sample. NHSA Dialog, $8(1), 72-87$.

Hu, L., \& Bentler, P. M. (1999). Cutoff criteria for fit indexes in covariance structure analysis: Conventional criteria versus new alternatives. Structural Equation Modelling: An Interdisciplinary Journal, 6(1), 1-55.

JASP Team (2019). JASP(Version 0.9.2) [Computer software].

Knowles, E. S., \& Byers, B. (1996). Reliability shifts in measurement reactivity: Driven by content engagement or self-engagement? Journal of Personality and Social Psychology, 70(5), 1080-1090.

Lam, T. C. M., Green, K. E., \& Bordignon, C. (2002). Effects of item grouping and position of "Don't know" option on questionnaire response. Field Methods, 14(4), 418-432.

Mahoney, J. L., Lord, H., \& Carryl, E. (2005). An ecological analysis of after-school program participation and the development of academic performance and motivational attributes for disadvantaged children. Child Development, 76(4), 811-825.

Moreland, J., Hendy, S., \& Brown, F. (2008). The validity of a personality disorder diagnosis for people with an intellectual disability. Journal of Applied Research in Intellectual Disabilities, 21(3), 219-226.

Pedhazur, E. J., \& Schmelkin, L. P. (1991). Measurement, design, and analysis: An integrated approach. NJ: Lawrence Erlbaum Associates.

Penketh, V., Hare, D. J., Flood, A., \& Walker, S. (2014). Attachment in adults with intellectual disabilities: Preliminary investigation of the psychometric properties of Manchester attachment scale-third party observational measure. Journal of Applied Research in Intellectual Disabilities, 27(5), 458-470. 
Porter, S. R., Whitcomb, M. E., \& Weitzer, W. H. (2004). Multiple surveys of students and student fatigue. New Directions for Institutional Research, 121, 63-73.

Rosseel, Y. (2012). Lavaan: An R package for structural equation modelling. Journal of Statistical Software, 48(2), 1-36.

Skoczeń, I., Rogoza, R., Maćkiewicz, M., Najderska, M., \& Cieciuch, J. (2018). Investigating the structural model of the strengths and difficulties questionnaire. European Journal of Psychological Assessment, 34(5), 312-320.

Steinberg, L. (1994). Context and serial-order effects in personality measurement: Limits on the generality of measuring changes the measure. Journal of Personality and Social Psychology, 66(2), 341-349.

Stone, L. L., Otten, R., Engels, R. C. M. E., Vermulst, A. A., \& Janssens, J. M. A. M. (2010). Psychometric properties of the parent and teacher versions of the strengths and difficulties questionnaire for 4- to 12-year-olds: A review. Clinical Child and Family Psychology Review, 13(3), 254-274.

Tasse, M. J., \& Havercamp, S. M. (2006). The role of motivation and psychopathology in understanding the IQ-adaptive behavior discrepancy. International Review of Research in Mental Retardation, 31, 231-259.

Torr, J. (2003). Personality disorder in intellectual disability. Current Opinion in Psychiatry, 16(5), 517-521.

Weisz, J. (1999). Cognitive performance and learned helplessness in mentally retarded persons. In E. Zigler \& D. Bennett-Gates (Eds.), Personality development in individuals with mental retardation (pp. 17-46). Cambridge: Cambridge University Press.
Yando, R., \& Zigler, E. (1971). Outerdirectedness in the problem-solving of institutionalized and noninstitutionalized normal and retarded children. Developmental Psychology, 4(2), 277-288.

Zigler, E. (1961). Social deprivation and rigidity in the performance of feebleminded children. Journal of Abnormal and Social Psychology, 62(2), 413-421.

Zigler, E. (1966). Research on personality structure in the retardate. International Review of Research in Mental Retardation, 1, 77-108.

Zigler, E. (1999). The individual with mental retardation as a whole person. In E. Zigler \& D. Bennett-Gates (Eds.), Personality development in individuals with mental retardation (pp. 1-16). Cambridge: Cambridge University Press.

Zigler, E. (2001). Looking back 40 years and still seeing the person with mental retardation as a whole person. In H. N. Switzky (Ed.), Personality and motivational differences in persons with mental retardation (pp. 3-55). New York: Taylor \& Francis Group.

Zigler, E. (2002). A veteran worker urges new renewed research on personality factors in mental retardation. Japanese Journal of Special Education, 39(6), 1-13.

Zigler, E., \& Balla, D. (1972). Developmental course of responsiveness to social reinforcement in normal children and institutionalized retarded children. Developmental Psychology, 6(1), 66-73.

Zigler, E., Bennett-Gates, D., Hodapp, R. M., \& Henrich, C. C. (2002). Assessing personality traits of individuals with mental retardation. American Journal on Mental Retardation, 107(3), 181-193.

Publisher's Note Springer Nature remains neutral with regard to jurisdictional claims in published maps and institutional affiliations. 